\title{
Applying a New Automated Perimetry Pattern Based on the Stimulus Distribution of the Multifocal ERG to Improve Structure-Function Investigation in Glaucoma
}

\author{
Lívia M. Brandão, ${ }^{1}$ Matthias Monhart, ${ }^{2}$ Andreas Schötzau, ${ }^{1}$ Anna A. Ledolter, ${ }^{3}$ \\ and Anja M. Palmowski-Wolfe ${ }^{1}$ \\ ${ }^{1}$ Ophthalmology, Basel University Hospital, Basel, BS, Switzerland \\ ${ }^{2}$ Carl Zeiss AG, Feldbach, Switzerland \\ ${ }^{3}$ Department of Ophthalmology, Medical University of Vienna, Vienna, Austria \\ Correspondence should be addressed to Lívia M. Brandão; livia.brandao@usb.ch
}

Received 21 February 2017; Revised 7 June 2017; Accepted 12 September 2017; Published 7 November 2017

Academic Editor: Suphi Taneri

Copyright (c) 2017 Lívia M. Brandão et al. This is an open access article distributed under the Creative Commons Attribution License, which permits unrestricted use, distribution, and reproduction in any medium, provided the original work is properly cited.

\begin{abstract}
Purpose. To validate a new automated perimetry pattern ( $\mathrm{mf103}$ pattern) for the investigation of retinal structure-function relationships in glaucoma in comparison to the standard G2 pattern and to relate either field's performance to optical coherence tomography (OCT). Methods. Automated perimetry data from the mfERG103 pattern were compared with the standard G2 pattern in glaucoma patients (18) and controls (15). The results of both (mean defect (MD) and mean sensitivity (MS)) were compared with optical coherence tomography (OCT): retinal nerve fiber layer (RNFL) thickness, macular thickness (mT), and ganglion cell analysis (GCIPL). Nine patients were followed up after one year. Results. G2 pattern and mf103 pattern did not differ significantly in MD or MS. The mf103 pattern associated significantly with more RNFL sectors in both MD and MS $(p<0.01$ and $p<0.05$, resp.). GCIPL thickness was not significantly associated with either SAP protocols. Both protocols remained comparable after one-year follow-up. Conclusions. G2 and mf103 pattern can both differentiate patients from controls with no significant difference in performance. RNFL thickness defects correlated better with mf103 than G2 with POAG. The mfERG-103 perimetry pattern can be used to establish structure-function correlations in glaucoma and may enable a more direct comparison with objective electrophysiological data.
\end{abstract}

\section{Introduction}

Glaucoma continues to be one of the leading causes of blindness worldwide [1], and structure-function correlations are currently investigated in an attempt to optimize performance [2-7]. This is important, for example, in some patients, standard automated perimetry (SAP) and, in others, optical coherence tomography (OCT) may be the first indicator of disease $[8,9]$. Thus, there is still not a single examination which can provide precise and definitive early diagnosis [10].
In spite of evidence that standard automated perimetry reveals abnormalities only after a significant amount of ganglion cells has been lost $[8,11]$, it is still largely used as the "gold standard" for glaucoma diagnosis and follow-up. Recently, alternative electrophysiological methods have been proposed as an alternative objective measurement of glaucomatous retinal dysfunction, such as the pattern ERG [12-14] and the multifocal electroretinogram (mfERG) [15-21]. Hood et al. [22] have attempted to improve the correlation between visual field examinations and the mfERG in 
glaucoma by implementing a visual field (Humphrey) based on the mfERG and found poor correlation between visual field sensitivity and the standard mfERG [23]. Since then, recent applications of the mfERG in glaucoma have successfully focused on augmenting the inner retinal contribution to the mfERG to increase its sensitivity by, for example, including global flash paradigms [15-21, 24, 25].

In order to facilitate structure-function analysis and to allow better comparison between a multifocal ERG paradigm (2-global flash mfERG which presents global flashes between the $m$-sequence stimulation) [16, 25-27] and SAP in future studies, we customized an automated perimetry pattern (mf103 pattern, Octopus, Haag-Streit) to directly relate to the $103 \mathrm{mfERG}$ stimulus grid. The perimetry results from the mf103 pattern were compared with those obtained using the G2 pattern. The results of both SAP methods were then compared to OCT findings in POAG and controls. In addition, nine patients were followed up after one year.

\section{Materials and Methods}

Eighteen POAG patients were recruited at the Glaucoma Service, Department of Ophthalmology, University of Basel. Additionally, 15 controls were recruited outside the clinical environment. Each subject underwent testing of bestcorrected visual acuity (BCVA), Goldmann applanation tonometry, slit-lamp examination of the anterior segment, fundus biomicroscopy, automated perimetry (both protocols), and OCT. All examinations were performed by one of the authors (LMB). Informed consent was obtained prior to inclusion in the study. The study followed the tenets of the Declaration of Helsinki and was approved by the regional Ethics Committee.

Inclusion criteria for patients were the presence of a glaucomatous optic disc associated with thinning in the neuroretinal rim of the RNFL in the OCT and a normal intraocular pressure, if needed, controlled by topical medication.

Individuals were excluded from the study in cases of systemic diseases such as diabetes or arterial hypertension, if they had had any type of ocular surgery previously, if they were using medication which could influence the normal physiology of the eye, or if refraction was greater than plus/minus 6 diopters.

All patients were asked to return after one year, but only nine patients agreed to repeat both SAP protocols and OCT.

2.1. Automated Perimetry. Patients were tested with the standard glaucoma G2 program and the customized mf103 pattern in Octopus 900 (Haag-Streit AG, Switzerland). Overall MD and mean sensitivity (MS) were calculated directly by the machine software and compared between the two protocols. For structure-function analysis, focal values were exported and clustered offline to create averages corresponding to the specific areas examined in the OCT. Reliability was ensured by including only examinations with a fixation loss under $33 \%$ as well as false-positive and falsenegative rates under $25 \%$.

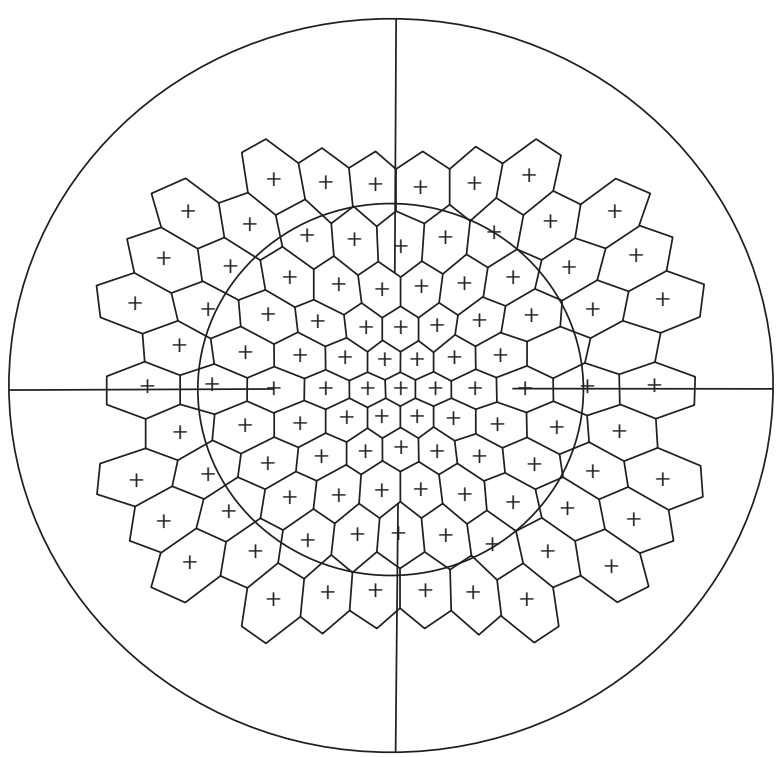

FIgURE 1: Superposition of the mf103 pattern and the mfERG stimulus grid (103 hexagons). The protocol has been developed to stimulate the individual areas, covering $50^{\circ}$, using the same test parameters as in standard perimetry testing: size III/3e; $100 \mathrm{~ms}$ duration; background $10 \mathrm{~cd} / \mathrm{m} 2 ; 0 \mathrm{~dB}$ equaled to $4000 \mathrm{asb}$.

2.2. Automated Perimetry-Customized mf103 Pattern. The mf103 pattern was customized based on the stimulus distribution of the 103 multifocal ERG (Figure 1). Coordinates were calculated by one of the authors (MM) so each visual field light stimulus would correspond exactly to the center of each hexagon from the mfERG stimulus grid, resulting in 103 stimuli covering the central $50^{\circ}$ of the retina. The mf103 pattern was tested as standard automated perimetry using a dynamic strategy with a white on white stimulus and was incorporated in the Octopus Perimeter 900. Thus, other than the different pattern of stimulation points, the same parameters applied as to the traditional glaucoma G2 protocol: stimuli size III, $100 \mathrm{~ms}$ duration, background $10 \mathrm{~cd} / \mathrm{m} 2,0 \mathrm{~dB}$ equaled to $4000 \mathrm{asb}$. Using the original Octopus dataset, the software was able to calculate all commonly used parameters (MD, MS, and SLV) for the specific points of the mf103 pattern, which will be referred to as MD103, MS103, and sLV103.

2.3. OCT. Images from the optic disc and the macula were obtained with the spectral optical coherence tomography $\left(\right.$ Cirrus SD-OCT $\left.{ }^{\mathrm{TM}}\right)$ using macular cube $(512 \times 128)$ and optic disc cube $(200 \times 200)$ protocols. Total macula thickness $(\mathrm{mT})$, ganglion cell-inner plexiform layer (GCIPL), and retinal nerve fiber layer (RNFL) thicknesses were calculated as per Cirrus software.

2.4. Structure-Function Analysis. For structure-function investigation, visual field stimulus points were clustered according to the $\mathrm{mT}$ and GCIPL areas of the OCT (Figure 2). For comparison to macular thickness, we analyzed the central $10^{\circ}\left(\mathrm{mT} 10,0-5^{\circ}\right.$ eccentricity) and $20^{\circ}$ (mT20, $0-10^{\circ}$ eccentricity) and for comparison to the GCIPL, 

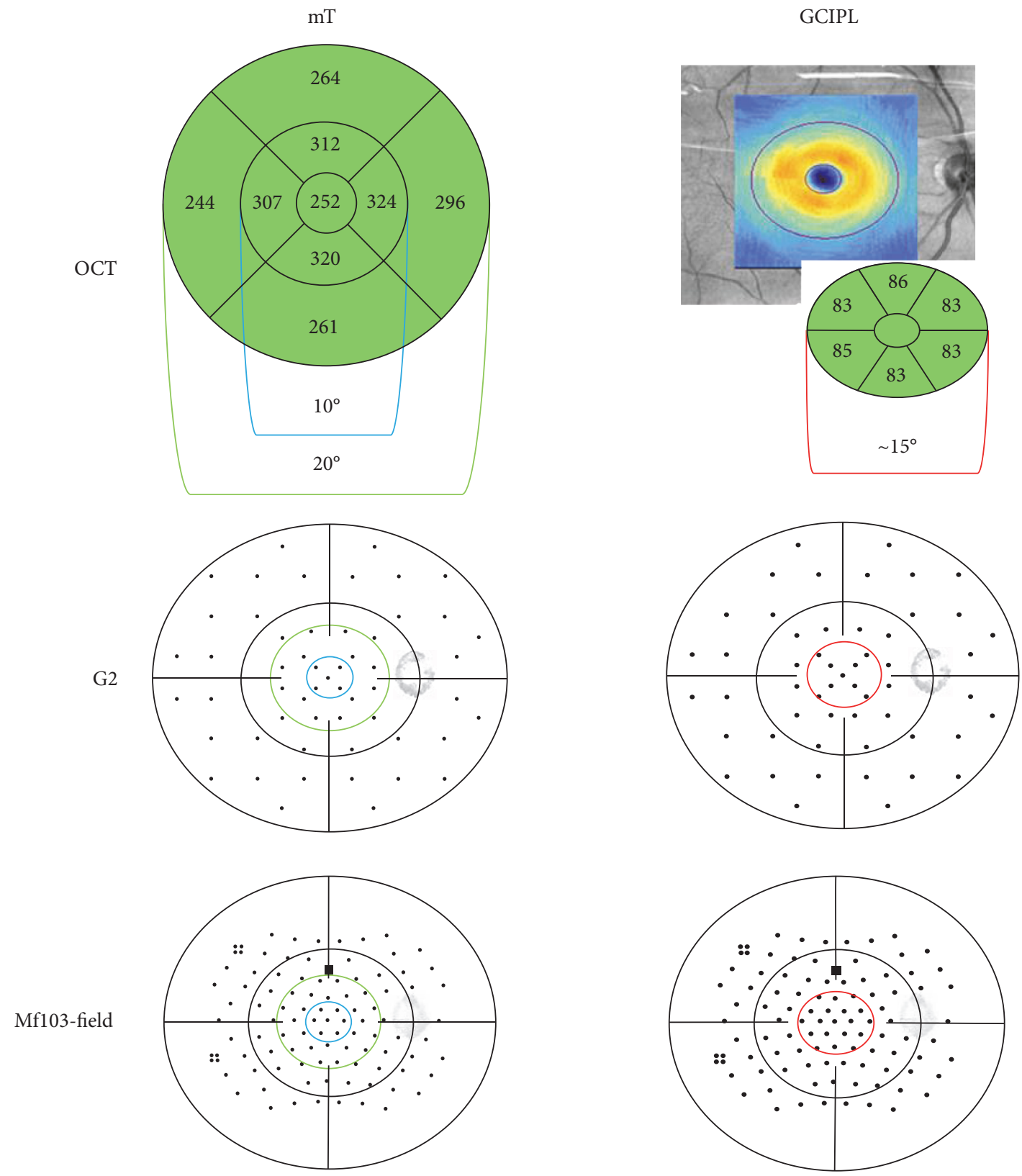

FIGURE 2: The group averages formed to compare OCT to visual field. The left column depicts the comparison between visual field and macular thickness, where the central $10^{\circ}$ ( $\mathrm{mT10}, 0-5^{\circ}$ eccentricity) and $20^{\circ}$ (mT20,0-10 eccentricity) were analyzed. The right column displays the comparison between visual field and the GCIPL in the central $\sim 15^{\circ}$ (GCIPL, $0-7.5^{\circ}$ eccentricity).

the central $\sim 15^{\circ}$ (GCIPL, $0-7.5^{\circ}$ eccentricity). Clustering of visual field points took into account the displacement of the retinal ganglion cells in the central 10 degrees, as described by Drasdo et al. [28] and by Hood et al. [29]. Therefore, $\mathrm{mT}$ values for the central $20^{\circ}$ and $10^{\circ}$ corresponded, respectively, to the central 17 and 5 points in G2 pattern and 31 points and 7 points in the $\mathrm{mfl} 103$ pattern protocol pattern. For comparison to GCIPL thickness measured within the central $15^{\circ}$, responses were averaged from the 9 central points of the G2 and the 19 central points from the mf103 pattern.

RNFL measurements from the optic disc are expressed in Cirrus analysis as 4 sectors (superior, inferior, temporal, and nasal) and 12-clock-hour sectors (Figure 3, top). For comparison to the RNFL sectors, focal visual field values were clustered into 12 corresponding group averages (retinal view), taking into account the nerve fiber distribution and its relation to Octopus stimulus points as published by Bürki and Monhart [30] (Figure 3, bottom).

Demographic data from all 33 participants are described in Table 1. Table 2 shows all OCT measures from both groups (mT, GCIPL, and RNFL).

2.5. Statistical Analysis. Demographic statistics were performed in SPSS (IBM, version 22). The statistical package $\mathrm{R}$ (version 3.0.2) was used to analyze the relationship between 

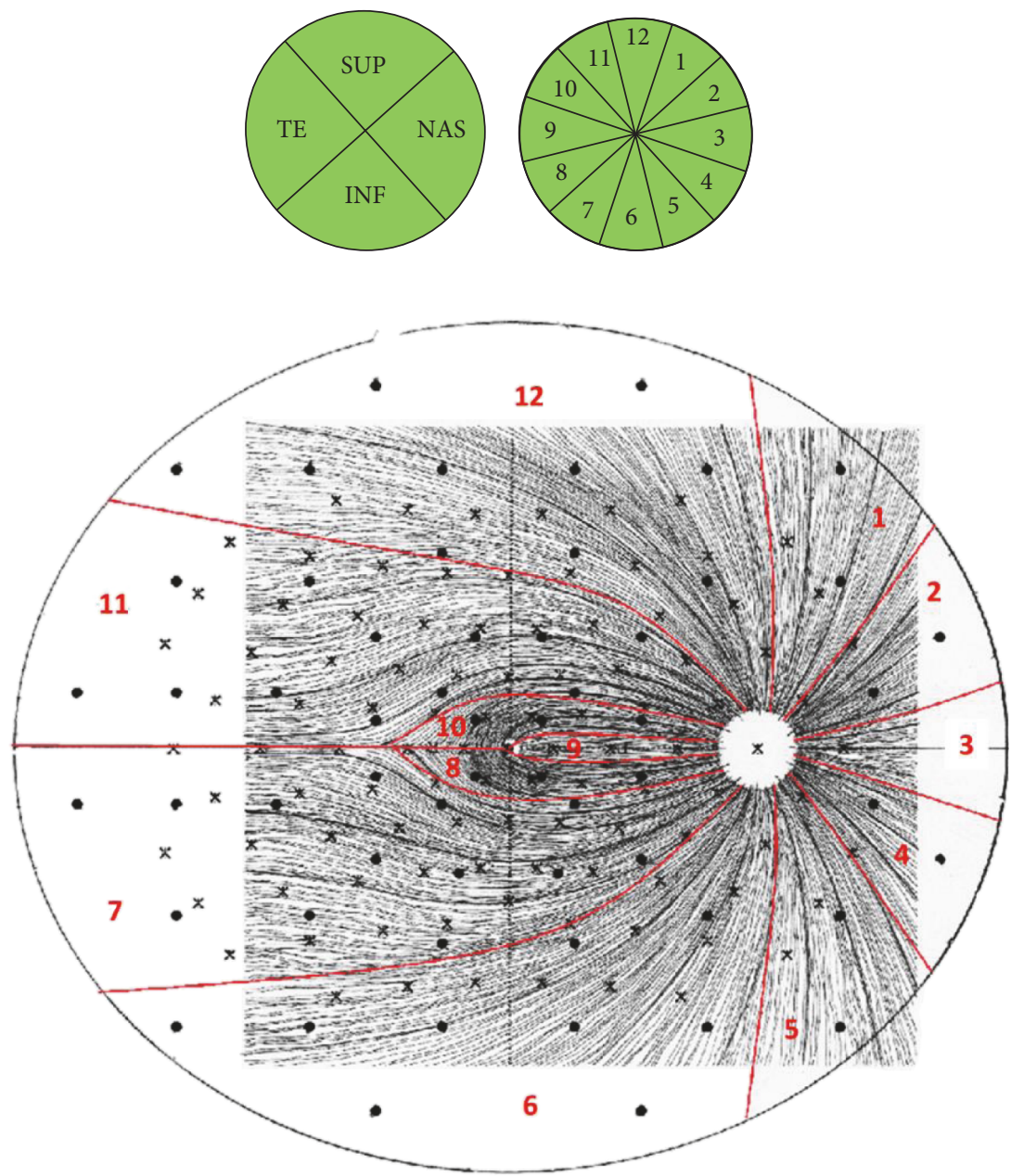

$\times$ MF103

- G2

Figure 3: The top row shows the RNFL distribution maps from the Cirrus ${ }^{\mathrm{TM}}$ RNFL thickness maps, for quadrants (left) and clock-hour sectors (right). The lower graph is an overlay of the G2 pattern (black dots) and the mf103 patterns (x) onto the RNFL distribution map. Red lines separate each of the 12 group averages formed to relate to the respective RNFL clock-hour sectors. G2 and mf103 group clusters are based on original RNFL and Octopus correlation maps created by Bürki and Monhart [30]. Sup: superior; NAS: nasal; INF: inferior; TE: temporal; 1 to 12: individual clock-hour sectors; "x" mf103; and "•": G2 pattern stimulus location.

TABLE 1: Participants' demographic and clinical variables.

\begin{tabular}{lccc}
\hline Group & POAG $(n=18)$ & Controls $(n=15)$ & $p$ value $^{*}$ \\
\hline Age (years) & $59.6 \pm 13.5$ & $49.2 \pm 7.2$ & $p=0.012$ \\
Sex (M/F) & $13 / 5$ & $4 / 11$ & \\
BCVA (decimal) & $0.9 \pm 0.0$ & $1.1 \pm 0.1$ & $p=0.001$ \\
IOP (mmHg) & $12.5 \pm 1.9$ & $13.2 \pm 2.7$ & $p=0.403$ \\
C/D ratio & $0.7 \pm 0.1$ & $0.2 \pm 0.0$ & $p=0.000$ \\
Duration (min) & & & \\
G2 & $5.6 \pm 2.0$ & $5.1 \pm 1.3$ & $p=0.436$ \\
mf103 & $8.5 \pm 1.7$ & $8.4 \pm 2.6$ & $p=0.924$ \\
G2 $\times$ mf103 & \multicolumn{2}{c}{$3.09( \pm 2.2)$} & $p<0.01$ \\
\hline
\end{tabular}

SD: standard deviation; BCVA: best-corrected visual acuity; IOP: intraocular pressure, if needed under use of topical medication; CDR: cup-to-disc ratio; ${ }^{*}$ Lavene's Test for equality of variances. exams using linear mixed effects models (adjusted to age and gender) and to compare the areas under the receiver operating characteristic curves (AUC). Results are presented as slope coefficients with corresponding $p$ values.

\section{Results}

On average, the G2 field testing required 5.35 minutes and the mf103 required 8.45 minutes. Thus, the mf103 pattern test was three minutes longer than the G2 pattern test. This was the same for glaucoma and control subjects. The specificity of both test patterns was $94.5 \%$. On average, the G2 pattern flagged $18.8 \%$ which is 11 of 59 test locations as abnormal. The mf103 pattern flagged $22.0 \%$ which is 23 of the 103 locations as abnormal. Therefore, the mf103 test showed a slightly higher sensitivity at the same specificity as compared to the G2 pattern. 
TABLE 2: Overview of the overall OCT measures $(\mu \mathrm{m})$ for POAG patients and controls.

\begin{tabular}{lcccc}
\hline Group & mT 10 & mT 20 & GCIPL & RNFL \\
\hline POAG $(n=18)$ & $297.2 \pm 13.2$ & $283.6 \pm 14.3$ & $67.9 \pm 8.9$ & $69.06 \pm 11.4$ \\
Controls $(n=15)$ & $307.5 \pm 12.4$ & $293.7 \pm 12.4$ & $80.9 \pm 4.8$ & $90.27 \pm 10.7$ \\
POAG versus controls & $p=0.029$ & $p=0.0013$ & $p<0.001$ & $<0.001$ \\
\hline
\end{tabular}

mT: macula thickness from the central $10^{\circ}\left(0^{\circ}-5^{\circ}\right.$ eccentricity) and the central $20^{\circ}\left({ }^{\circ} 0-10^{\circ}\right.$ eccentricity); GCIPL: ganglion cell-inner plexiform layer $\left(0^{\circ}-7.5^{\circ}\right.$ eccentricity) and RNFL: retinal nerve fiber layer thickness.

TABLE 3: In this comparison of the G2 and the mf103 pattern, the overall means and standard deviation $( \pm \mathrm{SD})$ values from both visual field patterns $(\mathrm{dB})$ are given for POAG and controls.

\begin{tabular}{|c|c|c|c|c|c|c|c|c|c|}
\hline Group & $\mathrm{MD}$ & & MD103* & MS & & MS103* & sLV & & sLV103* \\
\hline POAG $(n=18)$ & $3.47 \pm 4.0$ & $p-0.150$ & $3.95 \pm 3.8$ & $23.3 \pm 4.2$ & $p=0.297$ & $23.6 \pm 4.0$ & $4.6 \pm 2.7$ & $p=0.536$ & $4.8 \pm 2.9$ \\
\hline Controls $(n=15)$ & $0.13 \pm 1.7$ & $p=0.138$ & $0.59 \pm 1.5$ & $27.2 \pm 1.8$ & $p=0.310$ & $27.5 \pm 1.6$ & $2.0 \pm 0.5$ & $p=0.444$ & $2.2 \pm 0.6$ \\
\hline POAG versus controls & $p=0.0166$ & & $p=0.0026$ & $p=0.0152$ & & $p=0.0021$ & $p<0.001$ & & $p<0.001$ \\
\hline
\end{tabular}

For the G2 pattern, MD: mean defect; MS: mean sensitivity; and sLV: squared loss of variance; for the mf103 pattern, MD, MS, and sLV are marked with $103^{*} . p$ values are adjusted to age and gender. Italicized $p$ values compare the different field patterns, and upright $p$ values compare between POAG and control for each individual pattern.
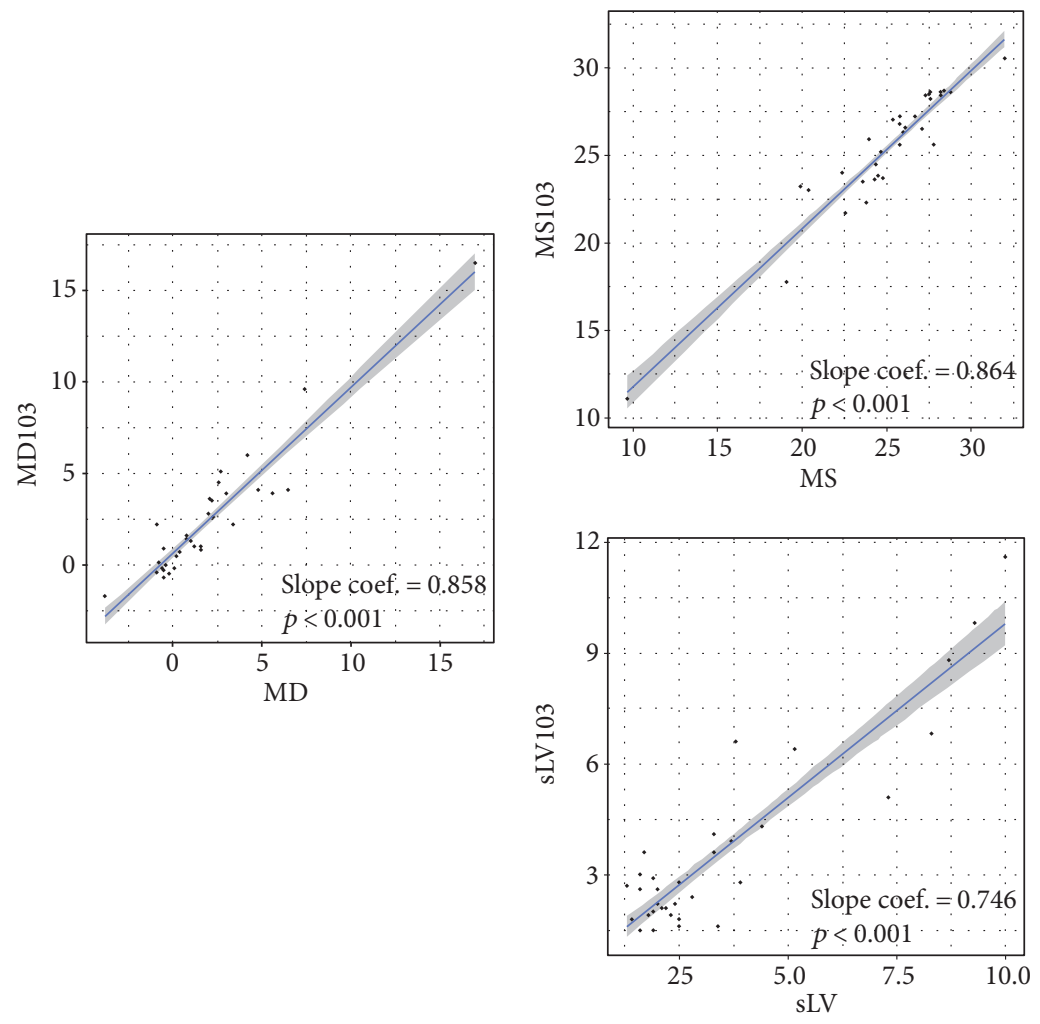

FIGURE 4: Linear mixed effects plots showing the significant positive relationship between G2 and mf103 patterns. For the G2 pattern, MD: mean defect; MS: mean sensitivity; and sLV: squared loss of variance; for the mf103 pattern, MD, MS, and sLV are marked with 103 . All values are in $\mathrm{dB}$.

Table 3 shows that overall MD and MS from both visual field protocols were able to significantly differentiate glaucoma from controls $(p<0.01)$. G2 and mf103 pattern did not differ significantly in overall MD, MS, or sLV.

Figure 4 shows a scatterplot of both visual fields depicting the significant positive relationship between both protocols for all parameters. When the performance was analyzed with the ROC curves, the AUC values did not differ significantly (DeLong test, Table 4).

In the central $10^{\circ}, \mathrm{MD}$ or MS did not differ significantly between POAG and control. Consistently, no patients had field defects ( $\geq 3$ adjacent points, $p<0.5 \%$ ) in the 
TABle 4: Area under the ROC curve (AUC) values of the G2 and the mf103 pattern. The $p$ values given in the right column compare the performance between the two field patterns applying the DeLong test.

\begin{tabular}{llc}
\hline & AUC & $p$ value \\
\hline MD & 0.837 & 0.878 \\
MD103 & 0.844 & \\
MS & 0.937 & 0.092 \\
MS103 & 0.856 & \\
sLV & 0.941 & 0.104 \\
sLV103 & 0.837 & \\
\hline
\end{tabular}

For the G2 pattern, MD: mean defect; MS: mean sensitivity; and sLV: squared loss of variance; for the mf103 pattern, MD, MS, and sLV are marked with 103.

central $10^{\circ}$ in either $\mathrm{G} 2$ or the $\mathrm{mf} 103$ pattern. In the central $15^{\circ}$ and $20^{\circ}$, MD was higher and MS was lower in POAG $(p<0.05)$. Three patients $(16.6 \%)$ had a defect in G2 inside the central $15^{\circ}$, while $4(22.2 \%)$ had a defect in mf103 pattern. For the central $20^{\circ}$, both visual field protocols identified 6 patients (33.3\%) with a defect. Figure 5 shows the Bland-Altman plots, that is, the difference against their mean for the two different field patterns and for the central $10^{\circ}, 15^{\circ}$, and $20^{\circ}$. The mean difference was close to zero with no evidence of bias. Furthermore, the shallow defects seen in our early glaucoma patients can be appreciated as there is a large overlap with controls at these eccentricities.

\subsection{Structure-Function Analysis}

3.1.1. $R N F L \times S A P$. The $\mathrm{mf103}$ pattern protocol had a significant association with more RNFL clusters than G2 for MD (negative) and MS (positive), in the quadrant sectors (Table 5) as well as in the clock-hour sectors (Figure 6). While there was agreement in the inferior quadrant, more superior and temporal sectors of the mf103 pattern showed a significant positive (MS103) and negative (MD103) association with RNFL thinning when compared to G2.

3.1.2. Macular Structure $\times S A P$. In control subjects, neither $\mathrm{mT}$ nor GCIPL was significantly associated with G2 or with mf103 pattern MD or MS within the central $20^{\circ}\left(10^{\circ}, 15^{\circ}\right.$, and $20^{\circ}$ ).

In POAG in the central $10^{\circ}$, the only significant association was between $\mathrm{mT}$ and MS (negative) as well as MD (positive) of G2 $(p<0.004)$. There was no significant association between $\mathrm{mT}$ and MS or MD of the mf103 pattern.

In POAG in the central $20^{\circ}$, the mf103 pattern showed a significant association between $\mathrm{mT}$ and $\mathrm{MD}$ (negative) as well as MS (positive) $(p<0.004)$. There was no significant association between $\mathrm{mT}$ and MS or MD of the complete G2 field.

Neither AP protocol was significantly associated with GCIPL thickness.

Figure 7 shows examples from individual patients. In these patients without central field defects, visual comparison demonstrates the superior correlation of the $\mathrm{mf} 103$ pattern to the RNFL thinning when compared to the G2.

3.2. Follow-Up. Nine POAG patients agreed to the follow-up evaluation at one year. Figure 8 shows the example of a patient at baseline and at one year. The $103 \mathrm{mf}$ pattern shows a clearer and consistent delineation of the arcuate field defect than the G2, where the second visual field obtained at one year might also be mistaken for a quadrant defect. Nonetheless, Table 6 shows that over the one year period, no significant changes had occurred in the glaucoma parameters: $\mathrm{MD}, \mathrm{MS}$, and sLV, IOP, $\mathrm{mT}$, and GCIPL. The significant correlation between $\mathrm{G} 2$ and $\mathrm{mf103}$ remained $(p<0.001)$.

\section{Discussion}

This study validates a customized visual field protocol (mf103 pattern) based on a multifocal electroretinogram (103 hexagons) pattern, for the assessment of patients with glaucoma.

The mf103 was comparable to the G2 in differentiating patients from controls. The slightly higher MD seen in the mf103 pattern might be caused by better coverage of the paracentral area and a higher frequency of affected test locations in that area. The sample size included in this study was not very large, and therefore a difference of $0.46 \mathrm{~dB}$ needs to be interpreted with care. It is however not due to an increased risk of false positives: The risk of more false positives (lower specificity) is not given, if, for example, the number of test locations that have to be at a certain probability level to consider the visual field as abnormal is calculated according to the number of test locations. For example, for a $\mathrm{G}$ visual field to be considered abnormal, one needs $>59 \times 0.05=3$ test locations at a $5 \%$ probability level and for the $\mathrm{mf103}$, one needs $>103 \times 0.05=5$ test locations at a $5 \%$ probability level. If this is considered, as done here, then sensitivity is not at the price of specificity.

OCT measures (mT, GCIPL, and RNFL) were significantly lower in our POAG patients. In our patient group, only the mf103 pattern showed a meaningful significant relation to $\mathrm{mT}$ where MD was increased with thinner $\mathrm{mT}$. The reverse was true for the G2 pattern. We cannot fully explain this discrepancy. One hypothesis could be the differences between SAP patterns in the location and the amount of testing points within the same tested area. Another influencing factor could be the variability in location of the SAP defect in our POAG population. Nevertheless, $\mathrm{mT}$ is outperformed by RNFL and GCIPL in glaucoma diagnostic, as conflicting results are observed, depending on stage of disease and type of glaucoma as reviewed by Wong et al. [31].

Surprisingly, neither SAP protocol correlated with GCIPL changes which may reflect the early stages of glaucoma tested, as observed by Lee et al. [32].

In regard to the RNFL, the significant relationship between RNFL sectors and SAP sensitivity in glaucoma based on the Hogan nerve fiber model has been already observed by Naghizadeh et al. [30,33]. In our study, we observed an improvement in this relationship when applying the mf103 pattern. Thus, the mf103 pattern may be a better tool in 


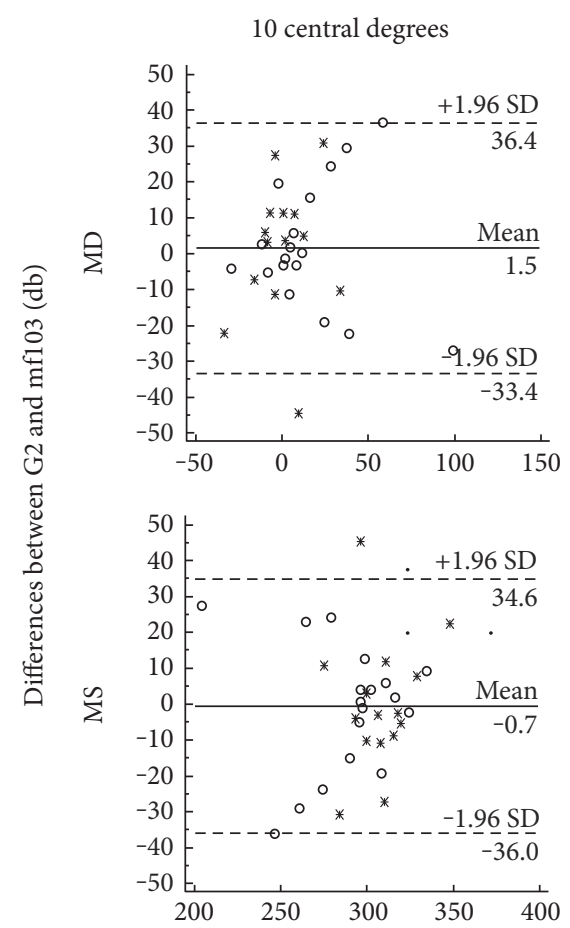

Group

- POAG

* Control
15 central degrees
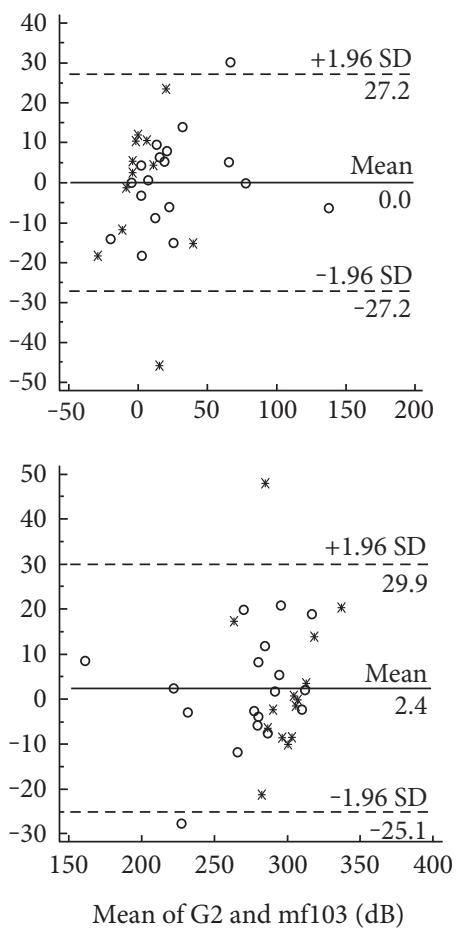

20 central degrees
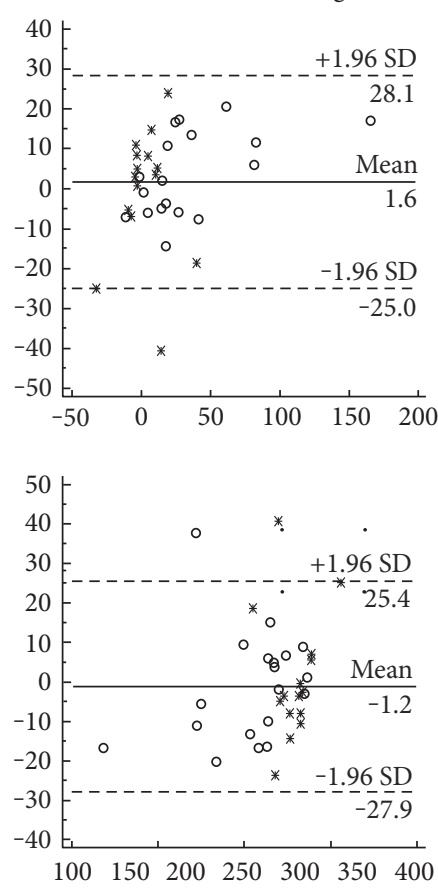

FIGURE 5: Bland-Altman plots (difference against mean) from MD and MS from the two different field patterns (G2 and mf103 pattern) for the central $10^{\circ}, 15^{\circ}$, and $20^{\circ}$. MD: mean defect; MS: mean sensitivity; G2: standard stimulus pattern form; mf103: customized mf103 pattern; both in Octopus Automated Perimetry; POAG: primary open-angle glaucoma group; SD: standard deviation.

TABLE 5: The relationship between OCT parameters and field parameters is summarized. Regression coefficients from the linear mixed effects analysis are given. The level of significance is depicted by “*” (* $p<0.01$ and $\left.{ }^{* *} p<0.001\right)$.

\begin{tabular}{lcccc}
\hline OCT & MD & MD103 & MS & MS103 \\
\hline RNFL & & & & \\
Average & $0.88^{*}$ & 0.27 & $-1.01^{*}$ & -0.67 \\
Superior & -0.21 & $-0.37^{* *}$ & 0.22 & $0.37^{*}$ \\
Nasal & 0.42 & -0.10 & -0.44 & 0.08 \\
Inferior & $-0.52^{* *}$ & $-0.77^{* *}$ & $0.56^{* *}$ & $0.81^{* *}$ \\
Temporal & 0.10 & $-0.64^{*}$ & -0.13 & $0.63^{*}$ \\
mT & & & & \\
$10^{\circ}$ & $0.53^{*}$ & 0.17 & $-0.52^{*}$ & -0.18 \\
$20^{\circ}$ & -0.19 & $-0.35^{*}$ & 0.19 & $0.35^{*}$ \\
GCIPL & 0.02 & -0.21 & -0.02 & 0.21 \\
\hline
\end{tabular}

For the G2 pattern, MD: mean defect and MS: mean sensitivity; for the mf103 pattern, MD and MS are marked with 103. OCT: RNFL: retinal nerve fiber layer thickness; mT: macula thickness from the central $10^{\circ}$ $\left(0^{\circ}-5^{\circ}\right.$ eccentricity), the central $20^{\circ}\left({ }^{\circ} 0-10^{\circ}\right.$ eccentricity $)$, and GCIPL: ganglion cell-inner plexiform layer $\left(0^{\circ}-7.5^{\circ}\right.$ eccentricity). glaucoma diagnosis, as the RNFL is supposed to be the most sensitive parameter in glaucoma diagnosis followed by GCIPL and $\mathrm{mT}$ [34].

In view of the increasing number of studies which use mfERG in glaucoma to detect glaucomatous dysfunction [15-18, 20, 21, 24-27, 35-37], the mf103 pattern is not just better in regard to structure-function correlation but also as a possibility of having a one-to-one direct correlation between visual field sensitivity and mfERG.

The mf103 pattern is based on the stimulus grid of the 103 hexagon mfERG. In view of structure-function analysis, more test points are included when the mf103 pattern is applied. In individual patients, this appears to better delineate arcuate defects (Figures 6 and 7) and overall, the mf103 pattern correlated better with RNFL thickness than the G2 pattern. This is in agreement with other studies which were able to identify visual field defects beyond the standard resolution from conventional perimetry in glaucoma, applying high spatial resolution perimetry [38-40]. In the nine patients that agreed to follow-up at one year, there was no significant difference in the changes observed in either field.

In this study, the mf103 pattern was programmed on the Octopus Perimeter 900 to exactly reflect the Veris multifocal ERG-103 hexagons scaling. Therefore, the results are not directly comparable to other patterns and 

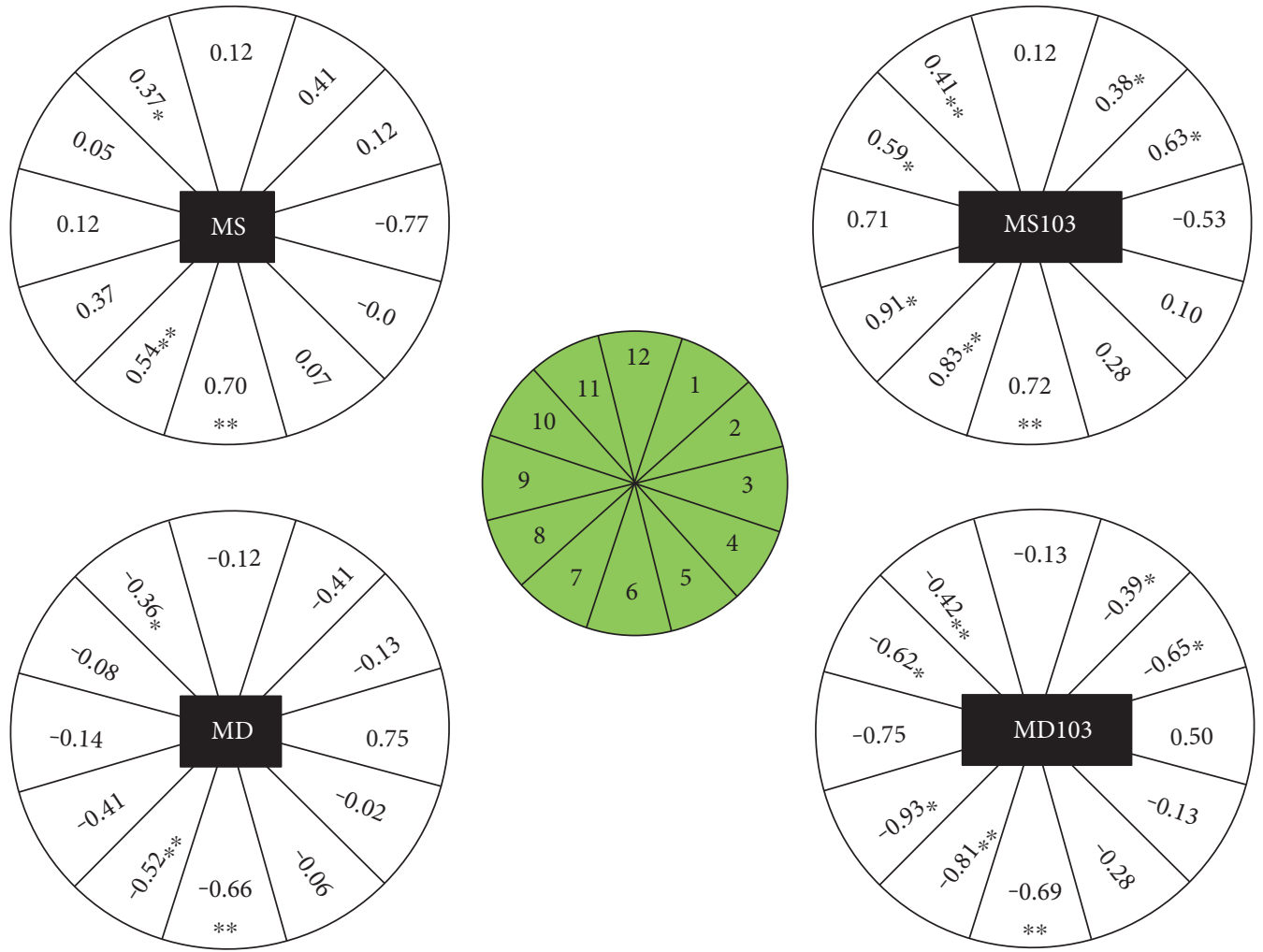

Figure 6: RNFL relationship to the G2 pattern (left) and to the mf103 pattern (right) is shown for the individual clock-hour sector (1 to 12 ). Numeric values are the regression coefficients from the linear mixed effects analysis. The level of significance is depicted by a “*” $\left({ }^{*} p<0.01\right.$ and $\left.{ }^{* *} p<0.001\right)$. For the G2 pattern, MD: mean defect; MS: mean sensitivity; for the mf103 pattern, MD and MS are marked with 103.
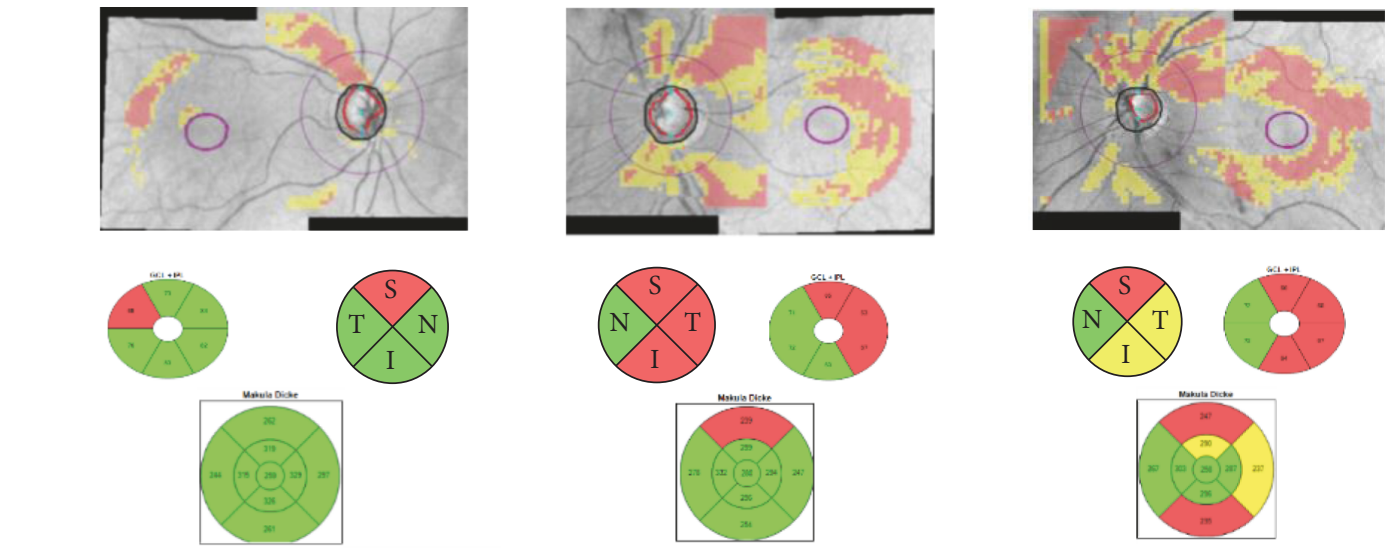

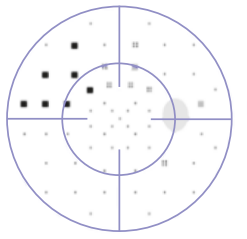

G2

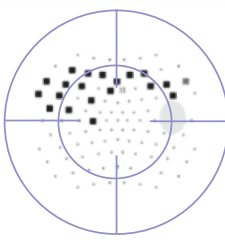

mf103

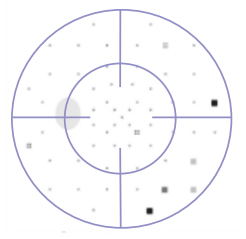

G2

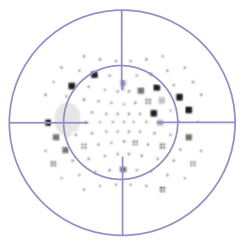

$\mathrm{mf103}$
G2

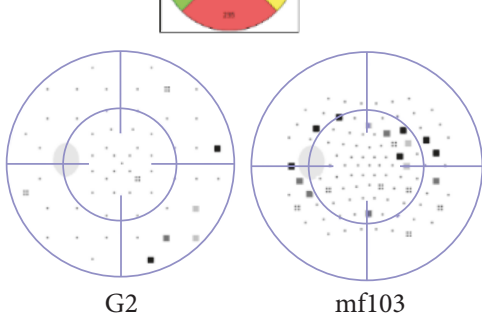

FIgURE 7: Graphic examples from 3 POAG patients showing the improved correlation between nerve-fiber-bundle defects and visual field parameters for the mf103 pattern compared to the G2 pattern. The top row is a composite of the RNFL and the GCIPL thickness deviation map taken from the Cirrus report. The middle row depicts significant deviations from normal (yellow and red sectors) for the RNFL (upper left), the GCIPL (upper right), and $\mathrm{mT}$ (bottom). The lower row shows the corresponding deviation maps for the G2 and the mf103 pattern. It is clear that the mf103 pattern has a higher resolution in the center where the G pattern under samples this field. 


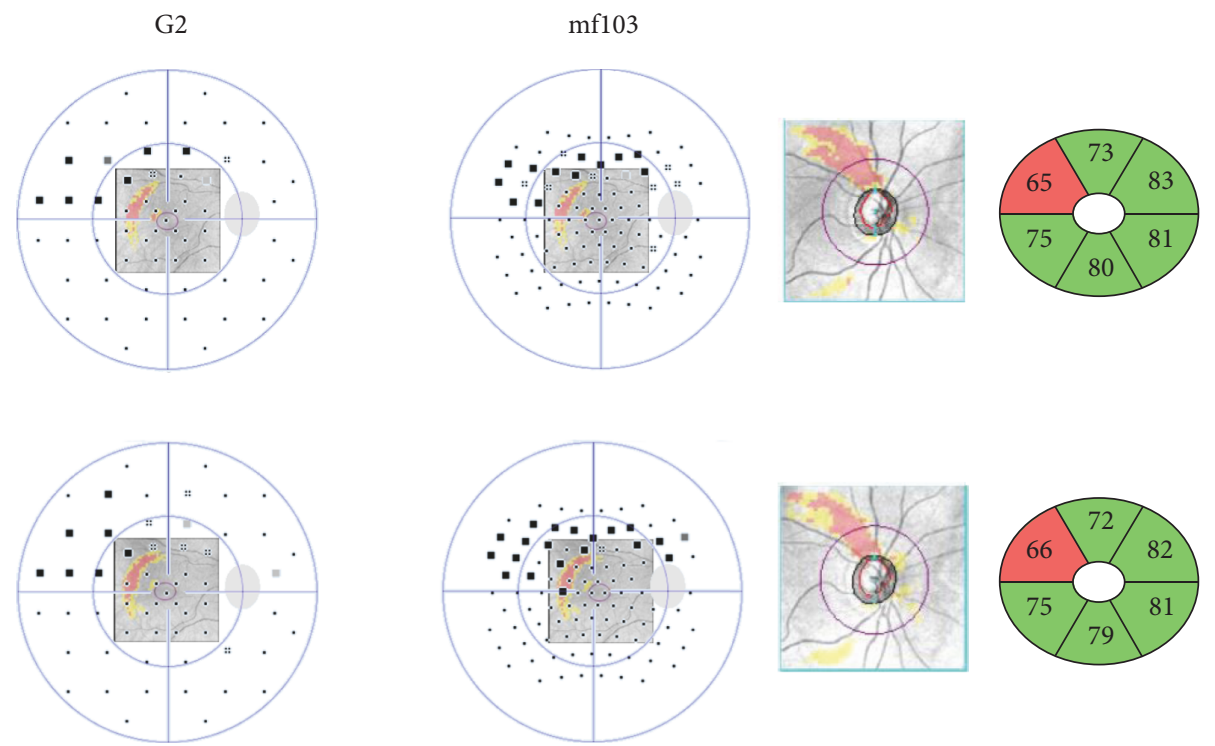

$(\%)$

$\begin{aligned} \therefore p>5 & \square p<1 \\ \therefore p<5 & \square p<0.5 \\ p<2 & \end{aligned}$

Figure 8: For the first patient in Figure 6, Figure 7 compares the findings at baseline (top) to the one-year follow-up (bottom). The left column shows the G2 pattern and the 2nd column, the mf103 pattern deviation plots as a composite with the GCIPL deviation map. The 3 rd column shows the RNFL deviation map and the rightmost column, the GCIPL thickness map as taken from the Cirrus analysis.

TABLE 6: Summary of the changes observed for the nine patients with a follow-up (FU) at 1 year. Changes in the means and standard deviation $(\mathrm{SD})$ values are given for visual field parameters $(\mathrm{dB})$ and OCT average thickness $(\mu)$ and also for IOP.

\begin{tabular}{lcccccccc}
\hline MD & MD103 & MS & MS103 & sLV & sLV103 & IOP & mT & GCIPL \\
\hline$\uparrow 0.02 \pm 1.7$ & $\downarrow 0.02 \pm 2.7$ & $\downarrow 0.08 \pm 1.7$ & $\uparrow 0.01 \pm 2.7$ & $\uparrow 0.06$ & $\uparrow 0.5$ & $\downarrow 0.33 \pm 2.5$ & $\downarrow 1.31 \pm 7.2$ & $\downarrow 0.35 \pm 0.6$ \\
$p=0.970$ & $p=0.981$ & $p=0.884$ & $p=0.991$ & $p=0.825$ & $p=0.286$ & $p=0.705$ & $p=0.603$ & $p=0.167$ \\
\hline
\end{tabular}

IOP: intraocular pressure in mmHg; For the G2 pattern, MD: mean defect; MS: mean sensitivity; for the mf103 pattern, MD and MS are marked with 103. mT: macula thickness; GCIPL: ganglion cell-inner plexiform layer thickness. $\uparrow$ marks an increase and $\downarrow$ marks a decrease at 1 year FU.

other manufacturer ERG devices. For those, the test would have to be reprogrammed according to manufacturer's scaling. If different patterns with different resolutions are applied, the resulting comparison to the G2 pattern needs to be evaluated, as it may differ.

In conclusion, G2 and mf103 pattern can both differentiate patients from controls with no significant difference in performance. The $\mathrm{mf} 103$ pattern was more sensitive in defect discrimination and in correlation to RNFL. Thus, the mf103 pattern can be applied in structure and function studies that include the mfERG without compromising sensitivity of the field examination.

\section{Ethical Approval}

All procedures performed in studies involving human participants were in accordance with the ethical standards of the institutional and/or national research committee and with the 1964 Helsinki Declaration and its later amendments or comparable ethical standards. This chapter does not contain any studies with animals performed by any of the authors.

\section{Consent}

All patients signed an informed consent approved by the Ethical Committee from Basel University Hospital before examination.

\section{Disclosure}

Funding organizations had no role in the design or conduct of this research. This work has been presented in parts at ARVO 2015/2016 and ISCEV/EUNOS 2015.

\section{Conflicts of Interest}

Matthias Monhart is an employee of Carl Zeiss AG (Feldbach, Switzerland) and has no financial interest in any of the methods implemented here. All other authors certify 
that they have no affiliations with or involvement in any organization or entity with any financial interest (such as honoraria, educational grants, participation in speakers' bureaus, membership, employment, consultancies, stock ownership or other equity interest, and expert testimony or patent-licensing arrangements) or nonfinancial interest (such as personal or professional relationships, affiliations, knowledge, or beliefs) in the subject matter or materials discussed in this manuscript.

\section{Acknowledgments}

This study was supported by AMP: Swiss National Fonds (SNF NMS 1823) and LHW Stiftung Liechtenstein.

\section{References}

[1] R. R. Bourne, H. R. Taylor, S. R. Flaxman et al., "Number of people blind or visually impaired by glaucoma worldwide and in world regions 1990 - 2010: a meta-analysis," PLoS One, vol. 11, no. 10, article e0162229, 2016.

[2] R. Malik, W. H. Swanson, and D. F. Garway-Heath, "'Structure-function relationship' in glaucoma: past thinking and current concepts," Clinical \& Experimental Ophthalmology, vol. 40, no. 4, pp. 369-380, 2012.

[3] D. C. Hood and R. H. Kardon, "A framework for comparing structural and functional measures of glaucomatous damage," Progress in Retinal and Eye Research, vol. 26, no. 6, pp. 688710, 2007.

[4] L. M. Ventura, N. Sorokac, R. De Los Santos, W. J. Feuer, and V. Porciatti, "The relationship between retinal ganglion cell function and retinal nerve fiber thickness in early glaucoma," Investigative Ophthalmology \& Visual Science, vol. 47, no. 9, pp. 3904-3911, 2006.

[5] R. S. Harwerth, A. S. Vilupuru, N. V. Rangaswamy, and E. L. Smith 3rd, "The relationship between nerve fiber layer and perimetry measurements," Investigative Ophthalmology \& Visual Science, vol. 48, no. 2, pp. 763-773, 2007.

[6] A. S. Raza, J. Cho, C. G. de Moraes et al., "Retinal ganglion cell layer thickness and local visual field sensitivity in glaucoma," Archives of Ophthalmology, vol. 129, no. 12, pp. 1529-1536, 2011.

[7] D. C. Hood, S. C. Anderson, M. Wall, A. S. Raza, and R. H. Kardon, "A test of a linear model of glaucomatous structure-function loss reveals sources of variability in retinal nerve fiber and visual field measurements," Investigative Ophthalmology \& Visual Science, vol. 50, no. 9, pp. 42544266, 2009.

[8] L. A. Kerrigan-Baumrind, H. A. Quigley, M. E. Pease, D. F. Kerrigan, and R. S. Mitchell, "Number of ganglion cells in glaucoma eyes compared with threshold visual field tests in the same persons," Investigative Ophthalmology \& Visual Science, vol. 41, no. 3, pp. 741-748, 2000.

[9] S. T. Takagi, Y. Kita, F. Yagi, and G. Tomita, "Macular retinal ganglion cell complex damage in the apparently normal visual field of glaucomatous eyes with hemifield defects," Journal of Glaucoma, vol. 21, no. 5, pp. 318-325, 2012.

[10] F. A. Medeiros and A. J. Tatham, "Structure versus function in glaucoma: the debate that doesn't need to be," Ophthalmology, vol. 123, no. 6, pp. 1170-1172, 2016.
[11] H. A. Quigley, G. R. Dunkelberger, and W. R. Green, "Retinal ganglion cell atrophy correlated with automated perimetry in human eyes with glaucoma," American Journal of Ophthalmology, vol. 107, no. 5, pp. 453-464, 1989.

[12] S. Ganekal, S. Dorairaj, and V. Jhanji, "Pattern electroretinography changes in patients with established or suspected primary open angle glaucoma," Journal of Current Glaucoma Practice, vol. 7, no. 2, pp. 39-42, 2013.

[13] M. Bach, A. S. Unsoeld, H. Philippin et al., "Pattern ERG as an early glaucoma indicator in ocular hypertension: a long-term, prospective study," Investigative Ophthalmology \& Visual Science, vol. 47, no. 11, pp. 4881-4887, 2006.

[14] V. Porciatti, B. Bosse, P. K. Parekh, O. A. Shif, W. J. Feuer, and L. M. Ventura, "Adaptation of the steady-state PERG in early glaucoma," Journal of Glaucoma, vol. 23, no. 8, pp. 494-500, 2014.

[15] A. M. Palmowski, R. Allgayer, and B. Heinemann-Vemaleken, "The multifocal ERG in open angle glaucoma - a comparison of high and low contrast recordings in high- and low-tension open angle glaucoma," Documenta Ophthalmologica, vol. 101, no. 1, pp. 35-49, 2000.

[16] A. M. Palmowski, R. Allgayer, B. Heinemann-Vernaleken, and K. W. Ruprecht, "Multifocal electroretinogram with a multiflash stimulation technique in open-angle glaucoma," Ophthalmic Research, vol. 34, no. 2, pp. 83-89, 2002.

[17] Y. Shimada, M. A. Bearse Jr., and E. E. Sutter, "Multifocal electroretinograms combined with periodic flashes: direct responses and induced components," Graefe's Archive for Clinical and Experimental Ophthalmology, vol. 243, no. 2, pp. 132-141, 2005.

[18] X. Luo, N. B. Patel, R. S. Harwerth, and L. J. Frishman, "Loss of the low-frequency component of the global-flash multifocal electroretinogram in primate eyes with experimental glaucoma," Investigative Ophthalmology \& Visual Science, vol. 52, no. 6, pp. 3792-3804, 2011.

[19] J. M. Miguel-Jimenez, L. Boquete, S. Ortega, J. M. RodriguezAscariz, and R. Blanco, "Glaucoma detection by wavelet-based analysis of the global flash multifocal electroretinogram," Medical Engineering \& Physics, vol. 32, no. 6, pp. 617-622, 2010.

[20] B. Fortune, M. A. Bearse Jr., G. A. Cioffi, and C. A. Johnson, "Selective loss of an oscillatory component from temporal retinal multifocal ERG responses in glaucoma," Investigative Ophthalmology \& Visual Science, vol. 43, no. 8, pp. 26382647, 2002.

[21] B. Fortune, L. Wang, B. V. Bui, G. Cull, J. Dong, and G. A. Cioffi, "Local ganglion cell contributions to the macaque electroretinogram revealed by experimental nerve fiber layer bundle defect," Investigative Ophthalmology \& Visual Science, vol. 44, no. 10, pp. 4567-4579, 2003.

[22] D. C. Hood, V. Greenstein, L. Frishman et al., "Identifying inner retinal contributions to the human multifocal ERG," Vision Research, vol. 39, no. 13, pp. 2285-2291, 1999.

[23] D. L. McCulloch, M. F. Marmor, M. G. Brigell et al., "ISCEV standard for full-field clinical electroretinography (2015 update)," Documenta Ophthalmologica, vol. 130, no. 1, pp. 1-12, 2015.

[24] X. Luo, N. B. Patel, L. P. Rajagopalan, R. S. Harwerth, and L. J. Frishman, "Relation between macular retinal ganglion cell/inner plexiform layer thickness and multifocal electroretinogram measures in experimental glaucoma," Investigative 
Ophthalmology \& Visual Science, vol. 55, no. 7, pp. 4512-4524, 2014.

[25] A. M. Palmowski-Wolfe, M. G. Todorova, S. Orguel, J. Flammer, and M. Brigell, "The 'two global flash' mfERG in high and normal tension primary open-angle glaucoma," Documenta Ophthalmologica, vol. 114, no. 1, pp. 9-19, 2007.

[26] S. A. Kramer, A. A. Ledolter, M. G. Todorova, A. Schotzau, S. Orgul, and A. M. Palmowski-Wolfe, "The 2-global flash mfERG in glaucoma: attempting to increase sensitivity by reducing the focal flash luminance and changing filter settings," Documenta Ophthalmologica, vol. 126, no. 1, pp. 57-67, 2013.

[27] A. A. Ledolter, M. Monhart, A. Schoetzau, M. G. Todorova, and A. M. Palmowski-Wolfe, "Structural and functional changes in glaucoma: comparing the two-flash multifocal electroretinogram to optical coherence tomography and visual fields," Documenta Ophthalmologica, vol. 130, no. 3, pp. 197209, 2015.

[28] N. Drasdo, C. L. Millican, C. R. Katholi, and C. A. Curcio, “The length of Henle fibers in the human retina and a model of ganglion receptive field density in the visual field," Vision Research, vol. 47, no. 22, pp. 2901-2911, 2007.

[29] D. C. Hood, A. S. Raza, C. G. de Moraes et al., "Initial arcuate defects within the central 10 degrees in glaucoma," Investigative Ophthalmology \& Visual Science, vol. 52, no. 2, pp. 940946, 2011.

[30] E. M. Bürki and M. Monhart, "Update Octopus-Perimetrie 2.Teil," Schweizerische Fachzeitschrift für augenärztliche Medizin und Technologie mit Mitteilungen SOG, vol. 2, no. 2, pp. 9-13, 2007.

[31] J. J. Wong, T. C. Chen, L. Q. Shen, and L. R. Pasquale, "Macular imaging for glaucoma using spectral-domain optical coherence tomography: a review," Seminars in Ophthalmology, vol. 27, no. 5-6, pp. 165-171, 2012.

[32] K. Lee, Y. H. Kwon, M. K. Garvin, M. Niemeijer, M. Sonka, and M. D. Abràmoff, "Distribution of damage to the entire retinal ganglion cell pathway: quantified using spectraldomain optical coherence tomography analysis in patients with glaucoma," Archives of Ophthalmology, vol. 130, no. 9, pp. 1118-1126, 2012.

[33] F. Naghizadeh, A. Garas, P. Vargha, and G. Hollo, "Structurefunction relationship between the octopus perimeter cluster mean sensitivity and sector retinal nerve fiber layer thickness measured with the RTVue optical coherence tomography and scanning laser polarimetry," Journal of Glaucoma, vol. 23, no. 1, pp. 11-18, 2014.

[34] C. K. Leung, W. M. Chan, W. H. Yung et al., "Comparison of macular and peripapillary measurements for the detection of glaucoma: an optical coherence tomography study," Ophthalmology, vol. 112, no. 3, pp. 391-400, 2005.

[35] N. V. Rangaswamy, W. Zhou, R. S. Harwerth, and L. J. Frishman, "Effect of experimental glaucoma in primates on oscillatory potentials of the slow-sequence mfERG," Investigative Ophthalmology \& Visual Science, vol. 47, no. 2, pp. 753-767, 2006.

[36] A. M. Palmowski and K. W. Ruprecht, "Follow up in open angle glaucoma. A comparison of static perimetry and the fast stimulation mfERG," Documenta Ophthalmologica, vol. 108, no. 1, pp. 55-60, 2004.

[37] A. M. O. S. Palmowski-Wolfe and M. G. Todorova, "Multifocal oscillatory potentials in the 'two global flash' mfERG in high and normal tension primary open-angle glaucoma,"
Journal of Clinical \& Experimental Ophthalmology, vol. 2, no. $167,2011$.

[38] D. C. Hood, M. Nguyen, A. C. Ehrlich et al., "A test of a model of glaucomatous damage of the macula with high-density Perimetry: implications for the locations of visual field test points," Translational Vision Science \& Technology, vol. 3, no. 3, p. 5, 2014.

[39] M. C. Westcott, A. I. McNaught, D. P. Crabb, F. W. Fitzke, and R. A. Hitchings, "High spatial resolution automated perimetry in glaucoma," The British Journal of Ophthalmology, vol. 81, no. 6, pp. 452-459, 1997.

[40] M. C. Westcott, D. F. Garway-Heath, F. W. Fitzke, D. Kamal, and R. A. Hitchings, "Use of high spatial resolution perimetry to identify scotomata not apparent with conventional perimetry in the nasal field of glaucomatous subjects," The British Journal of Ophthalmology, vol. 86, no. 7, pp. 761-766, 2002. 


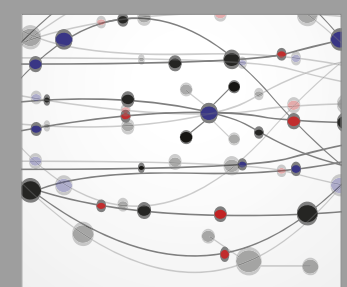

The Scientific World Journal
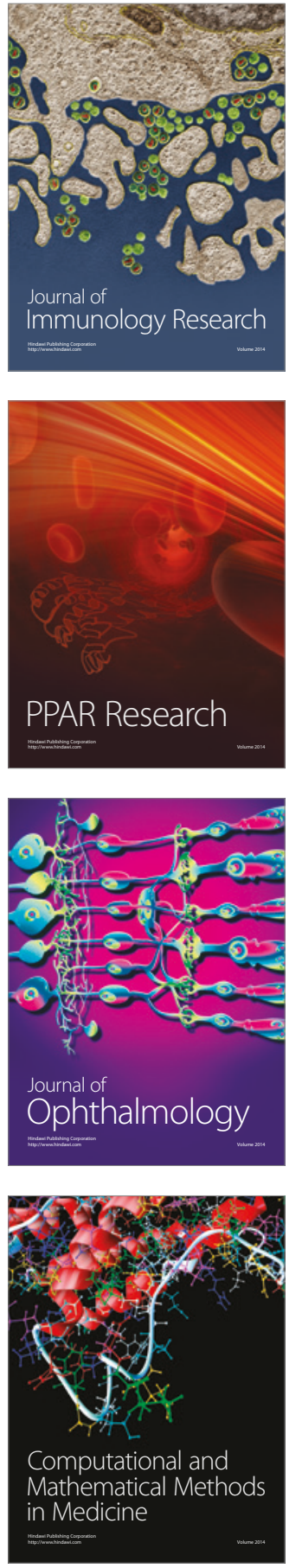

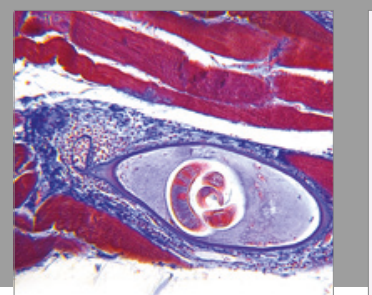

Gastroenterology Research and Practice
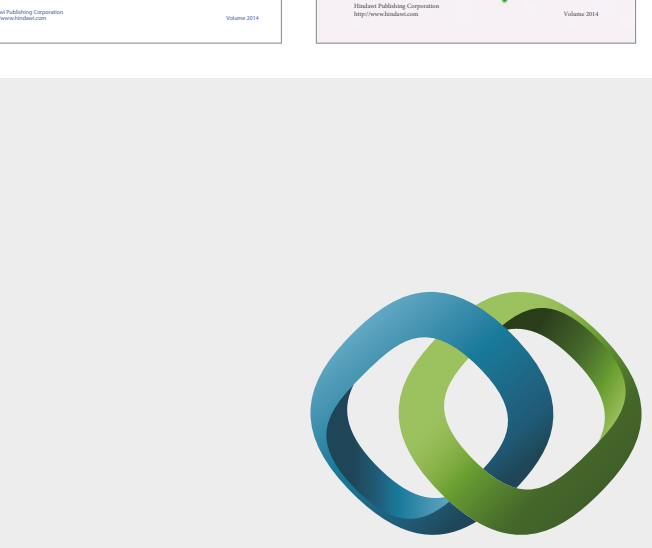

\section{Hindawi}

Submit your manuscripts at

https://www.hindawi.com
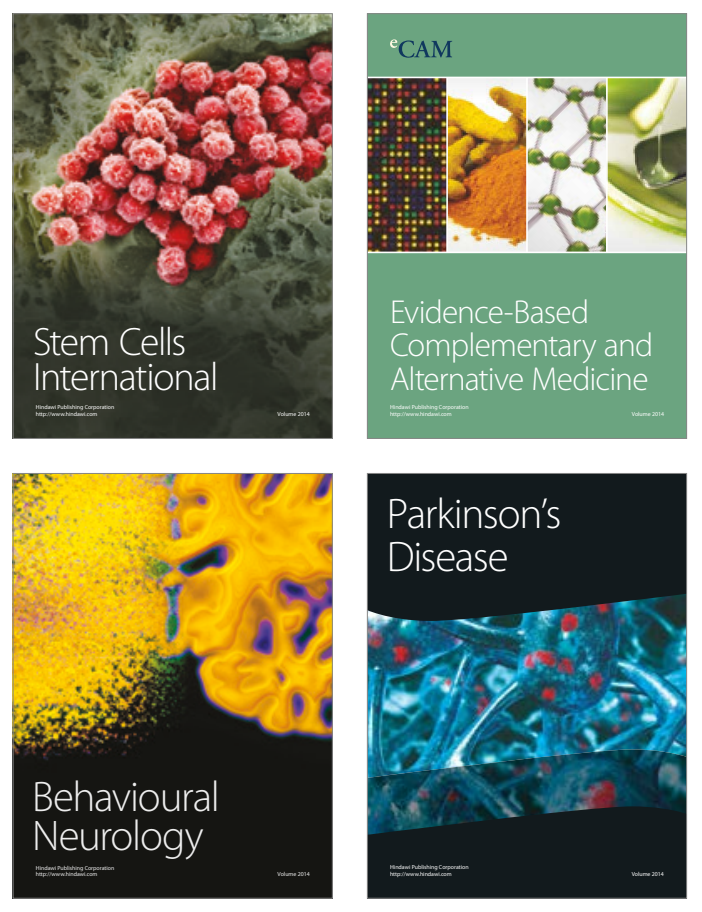
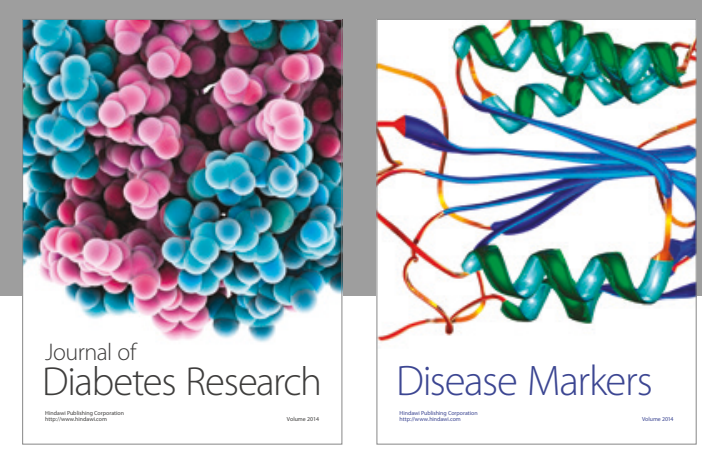

Disease Markers
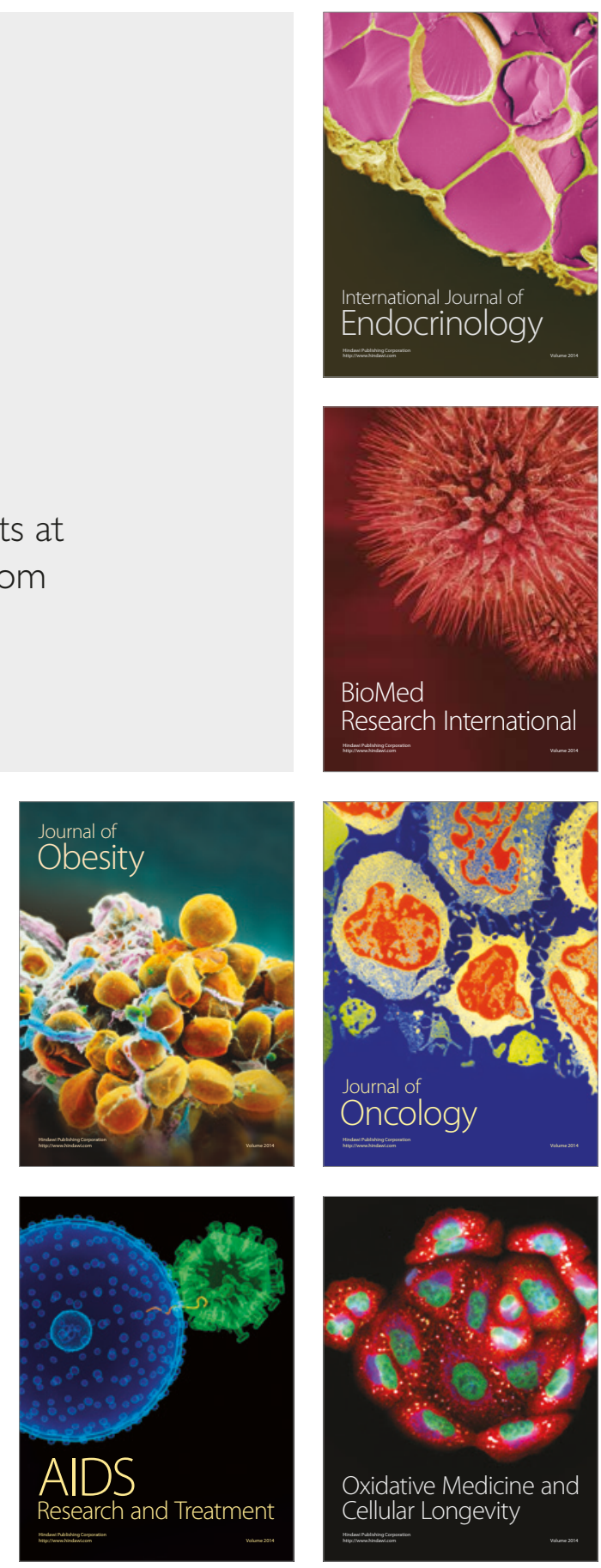